\title{
Report published on factors contributing to the spread of Campylobacter in the European Union
}

Eurosurveillance editorial team (eurosurveillance@ecdc.europa.eu) ${ }^{1}$

1. European Centre for Disease Prevention and Control, Stockholm, Sweden

Citation style for this article:

Eurosurveillance editorial team. Report published on factors contributing to the spread of Campylobacter in the European Union. Euro Surveill.

2010;15(33):pii=19641. Available online: http://www.eurosurveillance.org/ViewArticle.aspx?Articleld=19641

The European Food Safety Authority (EFSA) recently published a report on factors that may contribute to the spread of Campylobacter in live chickens and chicken carcasses. The findings of the report [1], based on an European Union (EU)-wide survey [2] will provide the basis for further work by scientific experts to investigate further how Campylobacter-contaminated chicken meat affects the levels of human campylobacteriosis.

The report states that it is about 30 times more likely that a Campylobacter-colonised broiler batch produces findings of sampled carcasses being contaminated with Campylobacter, compared with a non-colonised batch. Risks for contamination increase with the age of the slaughtered broilers as well as during certain months of the year. The contamination of carcasses with Campylobacter, higher Campylobacter counts on carcasses and Campylobacter colonisation of batches vary between countries and between slaughterhouses within countries, even when taking into account associated factors [1].

Over the last five years, campylobacteriosis is the most commonly reported zoonosis in the EU followed by salmonellosis and yersiniosis. The annual trends on the occurrence of zoonoses are reported on an annual basis in the joint European Centre for Disease Prevention and Control (ECDC) and EFSA annual report on zoonoses and food-borne outbreaks [3].

It is estimated that the handling, preparation and consumption of broiler meat may directly account for 20 to $30 \%$ of human cases of campylobacteriosis in the EU [4].

\section{References}

1. European Food Safety Authority (EFSA). Analysis of the baseline survey on the prevalence of Campylobacter in broiler batches and of Campylobacter and Salmonella on broiler carcasses, in the EU, 2008; Part B: Analysis of factors associated with Campylobacter colonisation of broiler batches and with Campylobacter contamination of broiler carcasses; and investigation of the culture method diagnostic characteristics used to analyse broiler carcass samples. EFSA Journal 2010; 8(8):1522. [132 pp.] doi:10.2903/j.efsa.2010.1522. Available from: http://www.efsa.europa.eu/en/efsajournal/ new.htm
2. Analysis of the baseline survey on the prevalence of Campylobacter in broiler batches and of Campylobacter and Salmonella on broiler carcasses in the EU, 2008, Part A: Campylobacter and Salmonella prevalence estimates. EFSA Journal 2010;8(03):1503). [99 pp.]. doi:10.2903/j. efsa.2010.1503. Available from: http://www.efsa.europa.eu/ en/efsajournal/scdoc/1503.htm

3. The Community Summary Report on Trends and Sources of Zoonoses, Zoonotic Agents and Food-borne Outbreaks in the European Union in 2008, EFSA Journal; 2010 8(1):1496. Available from: http://www.efsa.europa.eu/en/scdocs/ scdoc/1496.htm

4. EFSA Panel on Biological Hazards (BIOHAZ); Scientific Opinion on Quantification of the risk posed by broiler meat to human campylobacteriosis in the EU. EFSA Journal 2010; 8(1):1437. [89 pp.]. doi:10.2903/j.efsa.2010.1437. Available from: http:// www.efsa.europa.eu/en/efsajournal/scdoc/1437.htm 